\title{
Quantitative 7T Phase Imaging in Premanifest Huntington Disease
}

\author{
A.C. Apple, K.L. Possin, G. Satris, E. Johnson, J.M. Lupo, A. Jakary, K. Wong, D.A.C. Kelley, G.A. Kang, S.J. Sha, J.H. Kramer,
} M.D. Geschwind, S.J. Nelson, and C.P. Hess

\begin{abstract}
BACKGROUND AND PURPOSE: In vivo MR imaging and postmortem neuropathologic studies have demonstrated elevated iron concentration and atrophy within the striatum of patients with Huntington disease, implicating neuronal loss and iron accumulation in the pathogenesis of this neurodegenerative disorder. We used 7T MR imaging to determine whether quantitative phase, a measurement that reflects both iron content and tissue microstructure, is altered in subjects with premanifest Huntington disease.
\end{abstract}

MATERIALS AND METHODS: Local field shift, calculated from 7T MR phase images, was quantified in 13 subjects with premanifest Huntington disease and 13 age- and sex-matched controls. All participants underwent 3T and 7T MR imaging, including volumetric T1 and 7T gradient recalled-echo sequences. Local field shift maps were created from 7T phase data and registered to caudate ROIs automatically parcellated from the $3 \mathrm{~T} T 1$ images. Huntington disease-specific disease burden and neurocognitive and motor evaluations were also performed and compared with local field shift.

RESULTS: Subjects with premanifest Huntington disease had smaller caudate volume and higher local field shift than controls. A significant correlation between these measurements was not detected, and prediction accuracy for disease state improved with inclusion of both variables. A positive correlation between local field shift and genetic disease burden was also found, and there was a trend toward significant correlations between local field shift and neurocognitive tests of working memory and executive function.

CONCLUSIONS: Subjects with premanifest Huntington disease exhibit differences in 7T MR imaging phase within the caudate nuclei that correlate with genetic disease burden and trend with neurocognitive assessments. Ultra-high-field MR imaging of quantitative phase may be a useful approach for monitoring neurodegeneration in premanifest Huntington disease.

ABBREVIATIONS: $A U C=$ area under the curve; $C A G=$ cytosine-adenine-guanine; CAP $_{\mathrm{S}}=$ CAG-age-product scaled; eTIV = estimated total intracranial volume; $\mathrm{HD}=$ Huntington disease; LFS = local field shift; PLIC = posterior limb of the internal capsule; pmHD = premanifest Huntington disease; UHDRS = Unified Huntington's Disease Rating Scale

$\mathrm{H}$ untington disease (HD) is an autosomal dominant disorder caused by abnormal expansion of a CAG (cytosine-adenineguanine) nucleotide triplet within the Huntingtin gene, on the

Received September 16, 2013; accepted after revision January 9, 2014.

From the Departments of Radiology and Biomedical Imaging (A.C.A., J.M.L., A.J. S.J.N., C.P.H.) and Neurology (K.L.P., G.S., E.J., K.W., G.A.K., S.J.S., J.H.K., M.D.G.), University of California, San Francisco; and GE Healthcare (D.A.C.K.), Global Applied Sciences Laboratory, Menlo Park, California.

This work was funded in part by the University of California Discovery ITL-BIO0410148 , an academic-industry partnership grant with GE Healthcare, and by the National Institutes of Health grant 1S10RR026845-01.

Paper previously presented in part at: Annual Meeting of the International Society for Magnetic Resonance in Medicine, May 5-11, 2012; Melbourne, Australia.

Please address correspondence to Christopher P. Hess, MD, PhD, University of California, San Francisco, 505 Parnassus Ave, Room L-358, Box 0628, San Francisco, CA 94143-0628; e-mail: Christopher.Hess@ucsf.edu

- Indicates open access to non-subscribers at www.ajnr.org

$\checkmark \Delta$ Indicates article with supplemental on-line figure.

http://dx.doi.org/10.3174/ajnr.A3932 short arm of chromosome 4. ${ }^{1,2}$ The disease is characterized clinically by motor, cognitive, and behavioral symptoms and neuropathologically by degeneration across the brain, most prominently in the striatum. ${ }^{1,3}$ Genetic testing makes it possible to identify carriers before symptom onset, thereby opening a therapeutic window for interventions designed to delay or prevent the onset of disease. Currently, quantitative imaging of striatal volume is considered the most reliable method for monitoring progression in premanifest HD (pmHD). ${ }^{3-7}$ However, striatal volume is relatively insensitive, ${ }^{6,8,9}$ and additional markers are necessary to follow subclinical changes in clinical trials and to help define prognosis for the individual patient. ${ }^{8}$

Postmortem neuropathology and in vivo $1.5 \mathrm{~T}$ and $3 \mathrm{~T}$ MR imaging in $\mathrm{HD}$ have demonstrated increased iron in the caudate and putamen. ${ }^{10-14}$ As a recent example, Dumas et al ${ }^{15}$ used $3 \mathrm{~T}$ magnetic field correlation, a composite measure of iron levels, to compare subjects with early $\mathrm{HD}$, premanifest gene carriers, and 
healthy controls. Higher field correlation was found in patients with early HD compared with controls, but no difference was observed between the premanifest gene carrier group and controls. Among techniques sensitive to the presence of iron, MR phase imaging offers high contrast and permits numeric quantitation. ${ }^{16-19}$ Compared with 3T and lower field MR imaging, 7T phase imaging has higher signal-to-noise, heightened contrast and sensitivity, and improved visualization of anatomy. ${ }^{19-21}$ Together, these factors result in textural heterogeneity within the caudate that differs between matched controls and subjects with premanifest HD. ${ }^{22}$ In this study, we aimed to determine whether quantitative $7 \mathrm{~T}$ phase differs within the caudate nuclei between subjects with pmHD and healthy controls. Secondarily, we tested the association between caudate phase and volume and assessed relationships among phase and disease burden, severity of motor symptoms, and neurocognitive evaluation.

\section{MATERIALS AND METHODS Research Participants}

The University of California, San Francisco Committee on $\mathrm{Hu}-$ man Research provided institutional review board approval for this Health Insurance Portability and Accountability Act-compliant prospective study, and written consent was obtained from all subjects. Seventeen volunteers with genetically confirmed pmHD and 16 age- and sex-matched controls were recruited from a registry maintained by the UCSF Memory and Aging Center clinic or via their participation in other research projects at our institution between August 2011 and August 2013. Participants in the pmHD group tested positive for the HD mutation and had at least 40 CAG repeats. Three neurologists with expertise in HD (G.A.K., M.D.G., and S.J.S.) used the Unified Huntington's Disease Rating Scale (UHDRS) to determine the total motor score, between 0 and 124 , and the diagnostic confidence level, between 0 (healthy, no abnormalities) and 4 (motor abnormalities consistent with HD, $\geq 99 \%$ confidence), for each subject. Similar to prior studies of premanifest $\mathrm{HD},{ }^{23,24} 1$ subject who scored above 3 was excluded. Three other participants and 3 controls were excluded because they were unable to complete 7T MR imaging. Thirteen patients with pmHD and 13 controls were included for analysis.

\section{Disease Burden}

CAG-Age-Product Scaled $\left(\mathrm{CAP}_{\mathrm{S}}\right)$, an index developed in the PREDICT-HD study to approximate the time to HD diagnosis by using the age at motor onset and the number of CAG repeats, was calculated for each subject with $\mathrm{pmHD}$ as $\mathrm{CAP}_{\mathrm{S}}=$ Age $\times(\mathrm{CAG}-$ 33.66)/432.3326. ${ }^{23,25,26} \mathrm{CAP}_{\mathrm{S}}$ is classified as low $\left(0<\mathrm{CAP}_{\mathrm{S}}\right.$ $\leq 0.67)$, medium $\left(0.67<\mathrm{CAP}_{\mathrm{S}} \leq 0.85\right)$, or high $\left(\mathrm{CAP}_{\mathrm{S}}>0.85\right)$, reflecting higher cumulative disease burden and closer proximity to diagnosis. According to previously established norms, $\mathrm{CAP}_{\mathrm{S}}$ of less than, equal to, or greater than 1 indicates a 5 -year diagnosis probability of less than, equal to, or greater than $50 \%$, respectively.

\section{Cognitive Assessments}

Executive function and working memory are especially vulnerable to early brain changes in pmHD. ${ }^{27-29}$ We assessed cognitive function in these domains by using the National Institutes of
Health EXAMINER, a neuropsychological tool used to evaluate patients with pmHD in our clinical cohorts. ${ }^{30}$ The EXAMINER yields 4 scores: the overall Executive Composite score, the Working Memory score, the Cognitive Control score, and the Fluency score.

\section{MR Imaging Data Acquisition}

Subjects were scanned on 3T and 7T MR imaging scanners (GE Healthcare, Milwaukee, Wisconsin) in a single session by using a standardized protocol at both field strengths. Eight- and 32-channel head coils were used for 3T (GE Signa HDx 3T scanner) and 7T (GE MR950 7T scanner; a non-significant-risk investigational device), respectively. For $7 \mathrm{~T}$ phase imaging, a gradient recalledecho scan was acquired with $\mathrm{TR} / \mathrm{TE}=250 / 12.5 \mathrm{~ms}$, flip angle $=$ $15^{\circ}, \mathrm{FOV}=22 \mathrm{~cm}$, matrix $=1024 \times 768$, and $4-\mathrm{mm}$ section thickness with a 2-mm intersection gap for a scanning time of 6 minutes, 28 seconds. Volumetric T1-weighted images were also acquired at 7T by using an inversion recovery $\mathrm{T} 1$-weighted sequence with the following parameters, optimized empirically for gray-white contrast: TR/TE $=11 / 5 \mathrm{~ms}$, flip $=$ angle $20^{\circ}, \mathrm{FOV}=$ $22 \mathrm{~cm}$, matrix $=256 \times 256$, and $1-\mathrm{mm}$ section thickness for a duration of 3 minutes, 33 seconds. For accurate segmentation of caudate nuclei, volumetric T1-weighted imaging was also performed at $3 \mathrm{~T}\left(\mathrm{TR} / \mathrm{TE}=7 / 2 \mathrm{~ms}\right.$, flip angle $=15^{\circ}, \mathrm{FOV}=23 \mathrm{~cm}$, matrix $=256 \times 192$, 1-mm section thickness, 6 minutes, 18 second scanning time). A subspecialty certified neuroradiologist (C.P.H.) reviewed all images to assess whether subjective striatal atrophy was present in subjects with pmHD compared with controls.

\section{Image Processing and Analysis}

Magnitude and phase images were constructed from the multichannel 7T gradient recalled-echo data following the method of Hammond et al. ${ }^{31}$ Caudate ROIs were automatically delineated from the $3 \mathrm{~T}$ T1 data by using the subcortical segmentation algorithm $^{32}$ from Version 5.0.1 of the fMRI of the Brain Software Library image-processing package (FSL; http://www.fmrib.ox. ac.uk/fsl) (Fig 1A) and were normalized by estimated total intracranial volume (eTIV) in each subject. Using the T1 data from both field strengths as an intermediate for 6-parameter rigid-body registration, we aligned the caudate volumes with the $7 \mathrm{~T}$ images by using the FMRIB Linear Registration Tool in FSL (Fig $1 B) .^{33}$ eTIV was calculated from the $3 \mathrm{~T} \mathrm{~T} 1$ images by using in-house software written to implement the atlas-based method described by Buckner et al. ${ }^{34}$

White matter ROIs in the left and right posterior limb of the internal capsule (PLIC) were manually drawn for each subject on the $7 \mathrm{~T}$ phase images by using in-house software (Fig 1C). With the approach of Hammond et $\mathrm{al}^{20}{ }^{20}$ we used PLIC phase (recorded in hertz) to normalize phase measurements across the brain. Specifically, phase images were converted into quantitative maps of local field shift (LFS) by first subtracting the mean PLIC phase and then dividing by $\gamma \times \mathrm{B} 0$, measured in parts per billion at each pixel. Mean LFS was calculated within each caudate nucleus from the coregistered ROIs.

\section{Statistical Analysis}

Statistical analysis was performed using STATA, Version 13 (StataCorp, College Station, Texas). Groups were assessed for differences in sex by using $\chi^{2}$ tests for equality of proportions, and 

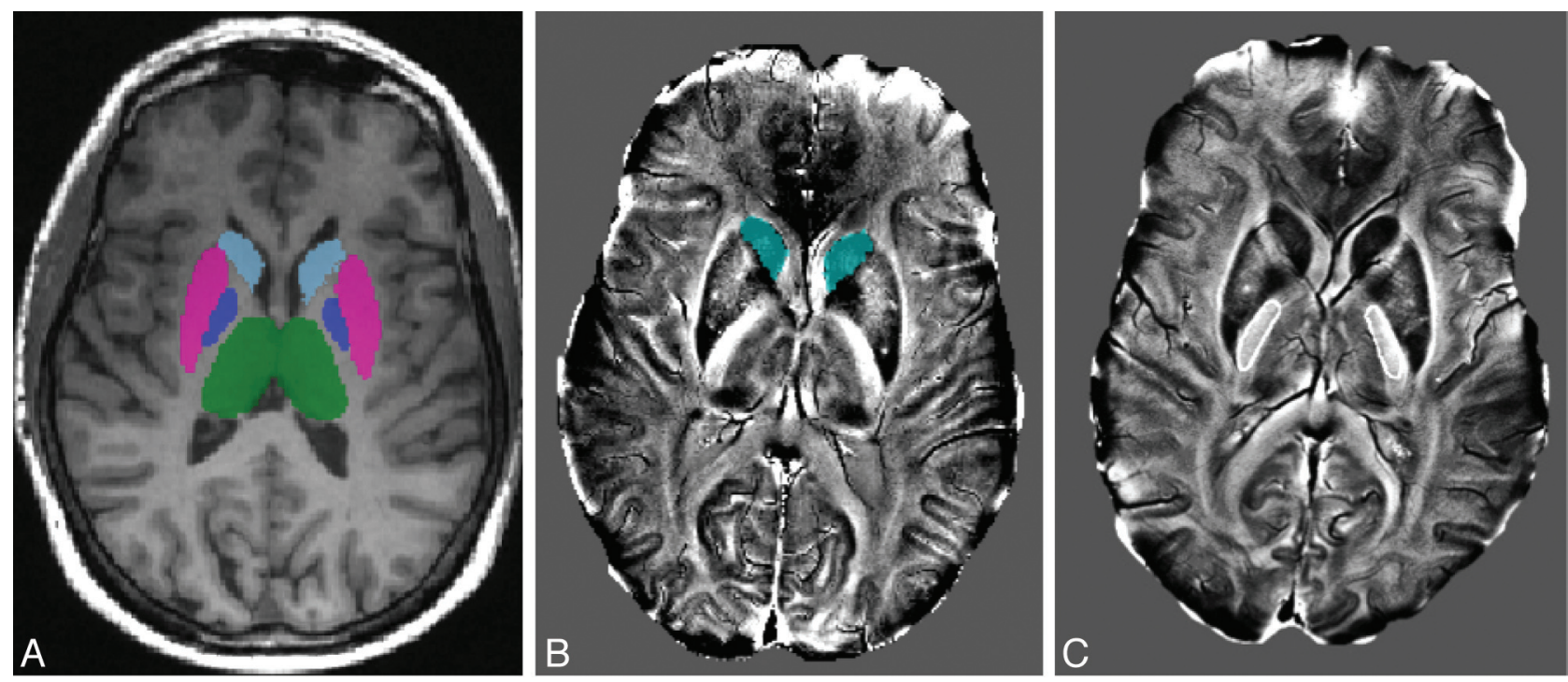

FIG 1. A, ROls are extracted automatically from 3T T1-weighted images by using FSL; the segmented caudate nuclei from 1 subject are shown in light blue. B, Caudate ROIs are automatically coregistered with the 7T data and overlaid onto the phase images. C, PLIC ROIs drawn manually (thin region drawn on each side of the brain in white) on 7T phase images. Mean PLIC phase was used for normalization in the construction of LFS maps in each patient.

1-way ANOVA was used to evaluate differences in age at the time of the MR imaging, years of education, and eTIV. Caudate volumes and mean LFS were compared across hemispheres by using $t$ tests for paired samples separately within each group. Because neither volume nor LFS departed significantly from Gaussian distributions based on tests of skewness and kurtosis, standard parametric analysis was used for all comparisons.

One-way ANCOVA was used to compare LFS and normalized volume between controls and subjects with pmHD, with age and sex included as covariates. Next, to investigate the relationship between LFS and normalized volume, we performed 2 separate analyses. First, Pearson partial correlation was applied, controlling for age, sex, and diagnosis. Second, logistic regression models were constructed to compare the accuracy of using LFS alone, normalized volume alone, and both values together as predictors to distinguish controls and subjects with pmHD. Finally, multivariate linear regressions were applied to evaluate relationships between LFS and CAP $_{\mathrm{S}}$ (disease burden), total motor UHDRS score, and EXAMINER score, by using sex as a covariate in the first case, age as a covariate in the second case, and both age and sex as covariates in the third case. For all tests, $P$ values $\leq .05$ were considered significant, adjusted for multiple comparisons with the Bonferroni correction in the regressions of LFS with EXAMINER scores.

\section{RESULTS}

\section{Subjects}

The Table summarizes baseline characteristics of subjects with pmHD and controls. There was no difference in age, sex, or eTIV between groups. A trend toward a greater number of years of education was seen in controls $(P=.077)$. Two subjects with pmHD fell into the low disease burden group; 4 , into the medium group, and 7, into the high group based on computed $\mathrm{CAP}_{\mathrm{S}}$ indices. The medium and low groups were combined for further statistical analyses. Total motor UHDRS scores for participants

\begin{tabular}{lccc}
\multicolumn{4}{l}{ Baseline characteristics for participants } \\
\hline & pmHD & Control & $P$ Value \\
\hline No. & 13 & 13 & - \\
Sex (female/male) & $7 / 6$ & $7 / 6$ & 1.00 \\
Age (yr) & $46.1 \pm 12.4$ & $45.7 \pm 15.3$ & 0.88 \\
Years of education & $15.7 \pm 2.5$ & $17.2 \pm 1.4$ & 0.077 \\
CAG repeats & $42.2 \pm 2.0$ & - & - \\
CAP $_{\mathrm{S}}$ & $0.867 \pm 0.153$ & - & - \\
UHDRS & $8.7 \pm 6.5$ & - & - \\
eTIV $\left(\mathrm{cm}^{3}\right)$ & $1465.1 \pm 137.5$ & $1431.0 \pm 71.4$ & 0.44 \\
\hline
\end{tabular}

Note:- - indicates not applicable.

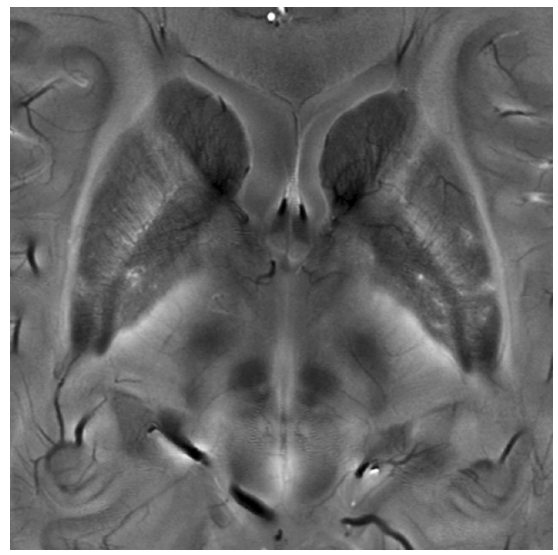

FIG 2. Magnified phase image centered on the basal ganglia in a representative subject with pmHD. The numeric values of phase reflect the composite effects of iron concentration, deoxyhemoglobin within veins, myelin, and tissue microstructure.

ranged from 0 to 21 . Visual inspection of 7T LFS maps confirmed that the caudate and PLIC boundaries were accurately delineated in each subject (Fig 2).

\section{Normalized Caudate Volume}

There was no difference between left and right normalized caudate volume in the pmHD or control groups; therefore, the mean 

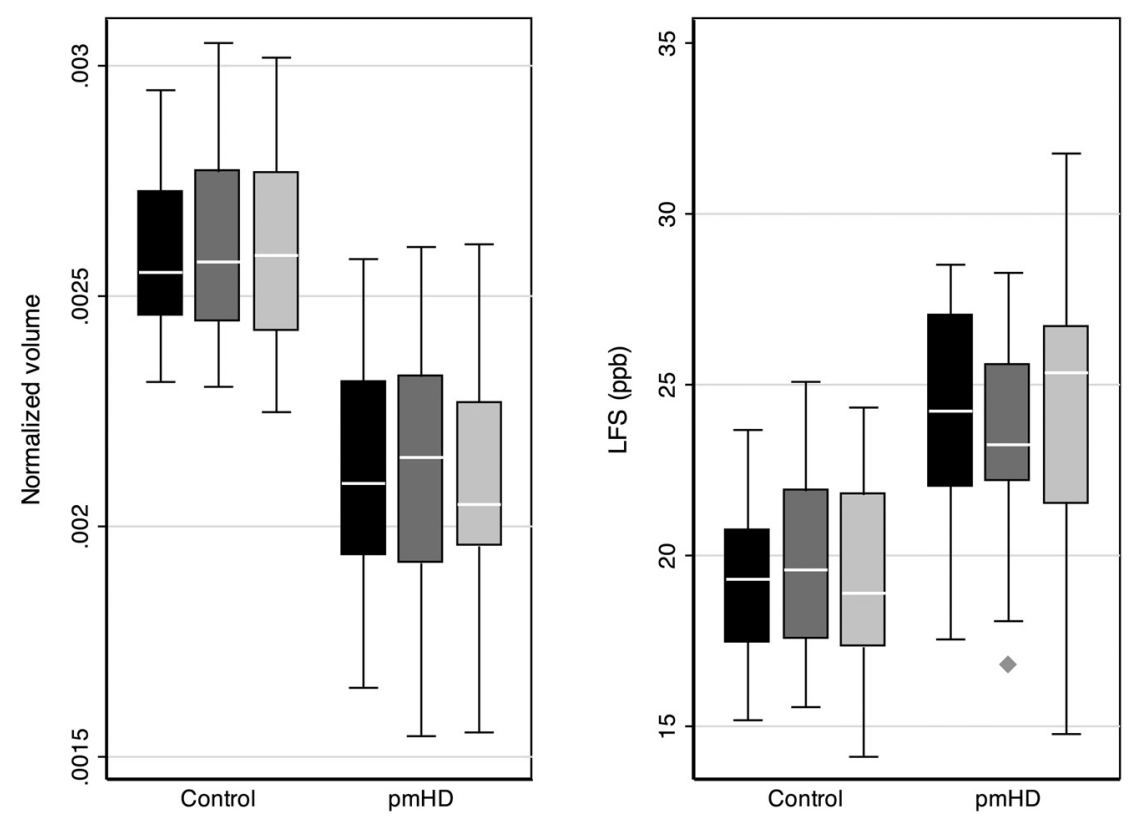

\section{Relationship between Caudate LFS and Disease Burden}

Figure 4 shows scatterplots illustrating the relationship between LFS and normalized caudate volume and $\mathrm{CAP}_{\mathrm{S}}$. In evaluating the relationship between LFS and these imaging and genetic markers of disease burden, partial correlations, controlled for age and sex, did not reveal an association between LFS and volume $(P=.42)$. In contrast, there was a strong correlation between LFS and $\operatorname{CAP}_{\mathrm{S}}\left(R^{2}=0.61, P=\right.$ $.003)$.

Logistic regression analyses showed that both LFS $(P=.008)$ and normalized volume $(P=.01)$ separately predicted disease state, as did bivariate modeling with both measurements $(P=.0004)$. There was no difference in the accuracy of the univariate models (area under the curve $[\mathrm{AUC}]=0.89$ [95\% CI, 0.77-1.0] for LFS, and $\mathrm{AUC}=0.92$ [95\% CI, 0.811.0] for normalized volume). Accuracy was higher for the bivariate logistic regression by using LFS and volume together $(\mathrm{AUC}=0.95[95 \% \mathrm{CI}, 0.88-1.0]$ ), implying that both variables contribute to the prediction of disease state. However, differences in AUC among the 3 models did not meet the criteria for significance, due to the small group sizes.

\section{Relationship between Caudate Measures and Total Motor UHDRS Score}

Controlled for age, partial correlations did not reveal an association between total motor UHDRS score and either normalized volume $(P=.97)$ or $\operatorname{LFS}(P=$ .63) (On-line Figure). (right) for subjects with pmHD and controls. In the left plot, triangles and circles refer to subjects with $\mathrm{pmHD}$ and controls, respectively. There was no correlation between caudate LFS and caudate volume. In the right plot, subjects in the low $(x)$, medium $(\square)$, and high $(\diamond) C_{C A P}$ groups. There is a strong positive correlation between $\mathrm{CAP}_{\mathrm{S}}$ and caudate $\mathrm{LFS}\left(R^{2}=0.61, P=.003\right)$.

\section{Relationship between Caudate LFS and Cognitive Assessments}

normalized volume for both nuclei was used for comparisons. Although visual inspection did not reveal disproportionate striatal atrophy, caudate volume was smaller $(P=.0001)$ in the pmHD group (Fig $3 A$ ). The difference in volume between the high and the medium-low $\mathrm{CAP}_{\mathrm{S}}$ groups was also significant $(P=.04)$. Age and sex did not influence this difference.

\section{Caudate Local Field Shift}

No difference between left and right caudate LFS was observed within either subjects with pmHD or controls; therefore, the mean LFS for both nuclei was used for comparisons. Subjects with pmHD had higher caudate LFS $(P=.0013)$ than controls (Fig $3 B)$. Although sex did not impact this difference, age was a significant covariate $(P=.024)$. A difference in LFS between the high and the medium-low $\mathrm{CAP}_{\mathrm{S}}$ groups was also found $(P=.03)$.
The results of multivariate regressions comparing LFS with EXAMINER scores for the pmHD and control groups are depicted in Fig 5. After Bonferroni correction, there was no correlation between LFS and EXAMINER scores when controlling for age and sex. There was, however, a trend toward correlation between LFS and Executive Composite score $\left(R^{2}=0.32, P=.031\right)$ and between LFS and Working Memory Factor $\left(R^{2}=0.35, P=.018\right)$.

\section{DISCUSSION}

The main result of this work is that measurements of LFS calculated from 7T gradient-echo phase MR imaging differ between subjects with pmHD and matched controls within the caudate nuclei. This result stands in contrast to prior work with ironsensitive techniques in $\mathrm{HD}$, which have shown elevated iron in the 

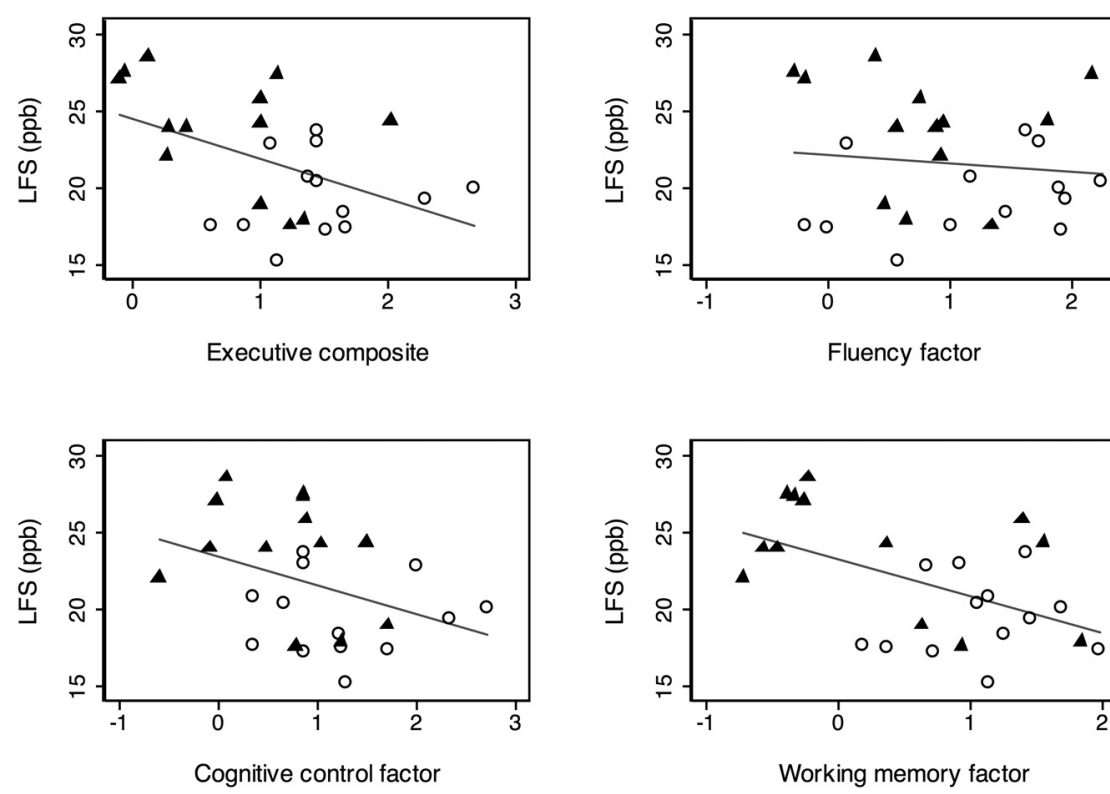

FIG 5. Scatterplots illustrating relationships between LFS and EXAMINER indices, including Executive Composite (top left), Fluency Factor (top right), Cognitive Control Factor (bottom left), and Working Memory Factor (bottom right). After correction for multiple comparisons, correlations were not significant but confirmed trends for Executive Composite $\left(R^{2}=0.32, P=\right.$ $.031)$ and Working Memory Factor $\left(R^{2}=0.35, P=.018\right)$. Circles $(O)$ and triangles $(\mathbf{\Delta})$ refer to controls and subjects with pmHD, respectively.

striatum of subjects with early- and late-stage HD but have not found increased iron in premanifest disease. ${ }^{15} \mathrm{We}$ also found that a positive caudate phase shift correlates with the genetic burden of disease and permits accurate prediction of disease state that improves when used together with normalized caudate volume. Finally, we determined that caudate LFS trends negatively with neuropsychological assessments of executive function and working memory, 2 cognitive domains previously reported to be affected when the National Institutes of Health EXAMINER is used in subjects with pmHD. ${ }^{35}$

In agreement with prior quantitative structural MR imaging studies, we found smaller caudate volume in subjects with premanifest HD. ${ }^{3-5,7}$ Similar to measurements of striatal volume, LFS can be quantitated semiautomatically to reduce interobserver variability and render analysis more feasible for large-scale clinical trials and research cohorts. PLIC measurements for normalizing phase were obtained manually in this study because fully automated analysis was not the focus of this work. However, templatebased techniques to define other ROIs, including the PLIC, could be similarly automated for fully automated quantitation.

Measurements of phase by using 7T MR imaging are modulated by a number of factors. Based on autopsy data derived from patients with late-stage $\mathrm{HD}^{10}$ and other MR imaging studies in $\mathrm{HD},{ }^{11,13,15}$ we hypothesize that the phase differences seen in this work primarily reflect abnormal deposition of iron. It is also possible that other magnetic susceptibility-shifting metabolites, including calcium, play a contributing role. The tissue magnetic architecture on ultra-high-field MR imaging is defined by the arrangement of normal proteins, lipids, and nonheme iron and other compounds at the cellular and subcellular level; disruptions in the normal arrangement of these molecular structures could also impact phase measurements. ${ }^{36}$ Compared with magnetic field correlation and phase imaging at lower field strengths, 7T has higher sensitivity to small alterations in tissue susceptibility and thus may enhance the ability to detect neuropathologic alterations related to HD before the onset of disease.

Age also impacts phase in many deep gray nuclei, including the caudate nuclei. $^{37,38}$ This effect was confirmed in the present work because age was found to be a significant covariate independent of disease status in multivariate analyses. Although we did not find that normalized caudate volume was impacted by age, it is recognized that the volumes of subcortical structures, including the caudate, decrease with advancing age, ${ }^{39}$ and the absence of this effect in the present study more likely reflects its limited power to detect small differences in caudate volume.

The absence of a correlation between caudate volume and LFS, together with the improved accuracy of delineating control and pmHD groups by using logistic regression with both variables simultaneously, suggests that these 2 measures reflect different pathologic end points of disease. This result is similar to that reported by Dumas et al, ${ }^{15}$ who did not observe a correlation between magnetic field correlation values and volume in their comparison of subjects with HD (both premanifest and manifest) and controls from the TRACK-HD study. Further work is necessary, however, to define the degree to which these 2 variables define different and overlapping dimensions of disease burden.

The strength of the correlation between LFS and $\mathrm{CAP}_{\mathrm{S}}$ implies that progressively higher phase shifts portend a shorter onset to clinically manifest disease. It is possible that alterations in phase indicate a greater magnitude of iron-dependent oxidative damage and neurotoxicity ${ }^{40}$ and thus provide a more direct predictor of disease onset than volume, which correlates largely to neuronal loss. The small number of subjects in this study precluded analysis of multiple brain regions; because of its known involvement in the earliest stages of $\mathrm{HD}$, we focused on the caudate. The fact that phase differences were detected between groups illustrates the high sensitivity of ultra-high-field MR imaging. In the future, studying phase in other parts of the brain may provide a broader picture of neurodegeneration in these patients.

Our study has several limitations. First, the number of subjects was small, and larger studies are necessary to delineate the strengths and limitations of using phase as a disease marker in HD. Second, because of the radio frequency-related intensity variation in the $7 \mathrm{~T}$ images, caudate volumes could not be reliably extracted from the 7T T1 data and a second 3T MR imaging was necessary for this purpose. With further technical refinement, we expect that both volumetric and phase measures can be reliably extracted from a single 7T examination. Furthermore, air-tissue interfaces and other sources of macroscopic susceptibility significantly distort 7T gradient-echo images and thereby make accu- 
rate phase measurement difficult. Although not prohibitive for studying the striatum, which is situated at some distance from most of these interfaces, the technique would currently be limited in parenchymal regions closer to the skull base or paranasal sinuses. Finally, the present work did not account for phase anisotropy in $7 \mathrm{~T}$ MR imaging. We anticipate that quantitative susceptibility mapping will mitigate the orientation dependence of phase contrast and further enhance the sensitivity of phase measurements to neurodegenerative pathology in patients with HD.

\section{CONCLUSIONS}

Quantitative measurements of 7T phase within the caudate nucleus in patients with pmHD differ from those in matched controls, most likely on the basis of differences in underlying tissue iron concentration. Because phase did not correlate with caudate volume and improved prediction of disease state when used together with caudate volume, we hypothesize that these measurements represent different pathologic endpoints of neurodegeneration in HD.

\section{ACKNOWLEDGMENTS}

The authors are grateful to the subjects who volunteered for this study.

Disclosures: Katherine L. Possin-RELATED: Grant: National Institute on Aging,* UNRELATED: Grants/Grants Pending: Michael J. Fox Foundation,* Hellman Family Foundation, ${ }^{*}$ Payment for Lectures (including service on Speakers Bureaus): SAFRA nursing course. Janine M. Lupo—RELATED: Grant: GE Healthcare, ${ }^{\star}$ Comments: We received grant support for scan time and the development of the acquisition and postprocessing techniques used in this study. However, this grant support has no impact on the results presented in this study. Angela Jakary-RELATED: Grant: University of California Discovery Grant, ${ }^{*}$ Comments: My staff research associate's salary was paid by Dr Sarah Nelson's University of California Discovery Grant (UCSF RAS System Award Number A115600) during the time that I contributed to the above article. Joel H. Kramer-UNRELATED: Consultancy: AbbVie, Royalties: Pearson Inc. Michael D. Geschwind-RELATED: Grant: BIO University of California Discovery Grant, * Comments: Funds obtained from the Department of Radiology and Biomedical Imaging supported the imaging for this study, UNRELATED: Consultancy: MEDACorp, The Council of Advisors, Guidepoint Global, Neurophage, Grants/Grants Pending: National Institutes of Health/High Q A104204 PREDICT-HD Study, ${ }^{*} \mathrm{Na}-$ tional Institutes of Health/National Institute on Aging (R01 AG-031189, R01 AG031189-6, K23 AG021989, AG031220), , Michael J Home Family Fund, * Tau Consortium, * National Institutes of Health/NCRR UCSF-CTSI (UL1 RR024131), ${ }^{*}$ National Institutes of Health/National Institute on Aging (AG021601), ${ }^{*}$ National Institutes of Health/National Institute of Neurological Disorders and Stroke contract (N01-NS-0-2328), ${ }^{*}$ contract number 12621 CHDI Inc, ${ }^{*}$ Travel/Accommodations/Meeting Expenses Unrelated to Activities Listed: HD Action Meeting, Comments: second meeting of Huntington Disease Clinics of California, Utah, and Nevada (HD Action) March 2-3, 2013. Airfare, lodging, meals, and ground transportation were provided by Lundbeck, Inc. Sarah J. Nelson-RELATED: Grant: National Institutes of Health (1S10RR02684501). This was an academic-industry partnership grant from the University of California, San Francisco.* Christopher P. Hess—RELATED: Grant: GE Healthcare, * Comments: funds for $7 \mathrm{~T}$ imaging and technical support. ${ }^{*}$ Money paid to the institution.

\section{REFERENCES}

1. Vonsattel JP, DiFiglia M. Huntington disease. J Neuropathol Exp Neurol 1998;57:369-84

2. LiSH, Li XJ. Huntingtin and its role in neuronal degeneration. Neuroscientist 2004;10:467-75

3. Bohanna I, Georgiou-Karistianis N, Hannan AJ, et al. Magnetic resonance imaging as an approach towards identifying neuropathological biomarkers for Huntington's disease. Brain Res Rev 2008;58:209-25

4. Aylward EH, Codori AM, Rosenblatt A, et al. Rate of caudate atrophy in presymptomatic and symptomatic stages of Huntington's disease. Mov Disord 2000;15:552-60
5. Rosas HD, Goodman J, Chen YI, et al. Striatal volume loss in HD as measured by MRI and the influence of CAG repeat. Neurology 2001;57:1025-28

6. Kipps CM, Duggins AJ, Mahant N, et al. Progression of structural neuropathology in preclinical Huntington's disease: a tensor based morphometry study. J Neurol Neurosurg Psychiatry 2005;76:650-55

7. Tabrizi SJ, Langbehn DR, Leavitt BR, et al. Biological and clinical manifestations of Huntington's disease in the longitudinal TRACK-HD study: cross-sectional analysis of baseline data. Lancet Neurol 2009;8:791-801

8. Weir DW, Sturrock A, Leavitt BR. Development of biomarkers for Huntington's disease. Lancet Neurol 2011;10:573-90

9. Rosas HD, Salat DH, Lee SY, et al. Cerebral cortex and the clinical expression of Huntington's disease: complexity and heterogeneity. Brain 2008;131:1057-68

10. Dexter DT, Carayon A, Javoy-Agid F, et al. Alterations in the levels of iron, ferritin and other trace metals in Parkinson's disease and other neurodegenerative diseases affecting the basal ganglia. Brain 1991;114:1953-75

11. Bartzokis G, Lu PH, Tishler TA, et al. Myelin breakdown and iron changes in Huntington's disease: pathogenesis and treatment implications. Neurochem Res 2007;32:1655-64

12. Bartzokis G, Tishler TA. MRI evaluation of basal ganglia ferritin iron and neurotoxicity in Alzheimer's and Huntingon's disease. Cell Mol Biol (Noisy-le-Grand) 2000;46:821-33

13. Bartzokis G, Cummings J, Perlman S, et al. Increased basal ganglia iron levels in Huntington disease. Arch Neurol 1999;56:569-74

14. Chen JC, Hardy PA, Kucharczyk W, et al. MR of human postmortem brain tissue: correlative study between $\mathrm{T} 2$ and assays of iron and ferritin in Parkinson and Huntington disease. AJNR Am J Neuroradiol 1993;14:275-81

15. Dumas EM, Versluis MJ, van den Bogaard SJ, et al. Elevated brain iron is independent from atrophy in Huntington's disease. Neuroimage 2012;61:558-64

16. Haacke EM, Mittal S, Wu Z, et al. Susceptibility-weighted imaging: technical aspects and clinical applications, part 1. AJNR Am J Neuroradiol 2009;30:19-30

17. Haacke EM, Cheng NY, House MJ, et al. Imaging iron stores in the brain using magnetic resonance imaging. Magn Reson Imaging 2005;23:1-25

18. Haacke EM, Gollapalli L, Rosas DH, et al. Using SWI as a means to better visualize the caudate nucleus in Huntington's disease. In: Proceedings of 14th Annual Meeting of the International Society for Magnetic Resonance in Medicine; Seattle, Washington. May 6-12, 2006:2657

19. Haacke EM, Ayaz M, Khan A, et al. Establishing a baseline phase behavior in magnetic resonance imaging to determine normal vs. abnormal iron content in the brain. J Magn Reson Imaging 2007;26:256-64

20. Hammond KE, Metcalf M, Carvajal L, et al. Quantitative in vivo magnetic resonance imaging of multiple sclerosis at 7 Tesla with sensitivity to iron. Ann Neurol 2008;64:707-13

21. Abduljalil AM, Schmalbrock P, Novak V, et al. Enhanced gray and white matter contrast of phase susceptibility-weighted images in ultra-high-field magnetic resonance imaging. J Magn Reson Imaging 2003;18:284-90

22. Doan NT, van den Bogaard SJA, Dumas EM, et al. Texture analysis of ultrahigh field $\mathrm{T}_{2}{ }^{*}$-weighted MR images of the brain: application to Huntington's disease. J Magn Reson Imaging 2014;39:633-40

23. Paulsen JS, Hayden M, Stout JC, et al. Preparing for preventive clinical trials: the Predict-HD study. Arch Neurol 2006;63:883-90

24. Harrington DL, Smith MM, Zhang Y, et al. Cognitive domains that predict time to diagnosis in prodromal Huntington disease. J Neurol Neurosurg Psychiatry 2012;83:612-19

25. Zhang Y, Long JD, Mills JA, et al. Indexing disease progression at study entry with individuals at-risk for Huntington disease. $A m J$ Med Genet B Neuropsychiatr Genet 2011;156B:751-63

26. Paulsen JS, Langbehn DR, Stout JC, et al. Detection of Hutington's 
disease decades before diagnosis: the PREDICT-HD study. J Neurol Neurosurg Psychiatry 2008;79:874-80

27. Duff K, Paulsen JS, Beglinger LJ, et al. "Frontal” behaviors before the diagnosis of Huntington's disease and their relationship to markers of disease progression: evidence of early lack of awareness. J Neuropsychiatry Clin Neurosci 2010;22:196-207

28. Hart E, Middelkoop H, Jurgens CK, et al. Seven-year clinical follow-up of premanifest carriers of Huntington's disease. PLoS Curr 2011;3:RRN1288

29. Stout JC, Paulsen JS, Queller S, et al. Neurocognitive signs in prodromal Huntington disease. Neuropsychology 2011;25:1-14

30. You SC, Geschwind MD, Sha SJ, et al. Executive functions in premanifest Huntington's disease. Mov Disord 2014;29(suppl 3):405-09

31. Hammond KE, Lupo JM, Xu D, et al. Development of a robust method for generating 7.0 $\mathrm{T}$ multichannel phase images of the brain with application to normal volunteers and patients with neurological diseases. Neuroimage 2008;39:1682-92

32. Patenaude B, Smith SM, Kennedy DN, et al. A Bayesian model of shape and appearance for subcortical brain segmentation. Neuroimage 2011;56:907-22

33. Jenkinson M, Bannister P, Brady M, et al. Improved optimization for the robust and accurate linear registration and motion correction of brain images. Neuroimage 2002;17:825-41
34. Buckner RL, Head D, Parker J, et al. A unified approach for morphometric and functional data analysis in young, old, and demented adults using automated atlas-based head size normalization: reliability and validation against manual measurement of total intracranial volume. Neuroimage 2004;23:724-38

35. You C, Satris G, Apple A, et al. New sensitive measures of executive functioning in premotor Huntington's disease. Neurology 2013; 80:S20.002

36. He X, Yablonskiy DA. Biophysical mechanisms of phase contrast in gradient echo MRI. Proc Natl Acad Sci U S A 2009;106:13558-63

37. Bilgic $B, P$ fefferbaum A, Rohlfing $T$, et al. MRI estimates of brain iron concentration in normal aging using quantitative susceptibility mapping. Neuroimage 2012;59:2625-35

38. Hagemeier J, Dwyer MG, Bergsland N, et al. Effect of age on MRI phase behavior in the subcortical deep gray matter of healthy individuals. AJNR Am J Neuroradiol 2013;34:2144-51

39. Murphy DG, DeCarli C, Schapiro MB, et al. Age-related differences in volumes of subcortical nuclei, brain matter and cerebrospinal fluid in healthy men as measured with magnetic resonance imaging. Arch Neurol 1992;49:839-45

40. Berg D, Youdim MB. Role of iron in neurodegenerative disorders. Top Magn Reson Imaging 2006;17:5-17 